\title{
Circle criterion observer for a compression system
}

\author{
Bjrnar Bhagen \\ Department of Engineering Cybernetics \\ Norwegian University of Science and Technology \\ Email: bjornar.bohagen@itk.ntnu.no
}

\author{
Jan Tommy Gravdahl \\ Department of Engineering Cybernetics \\ Norwegian University of Science and Technology \\ Email: tommy.gravdahl@itk.ntnu.no
}

\begin{abstract}
Observers for a compression system using turbo compressors are derived for a model that captures the phenomena of surge. Both a full order observer for pressure, mass flow and impeller speed and a reduced order observer for mass flow are presented. The observers assume measurements of pressures and impeller speed. Results are validated by simulations.
\end{abstract}

\section{Motivation}

Compression systems using turbo compressors, centrifugal or axial, are exposed to the phenomenons of surge and rotating stall. Surge is an axisymmtrical oscillation of the flow through the system, and is recognized by oscillations in system states such as mass flow and pressure. Rotating stall is a phenomena appearing locally in the compressor, and is characterized by the circumferential flow pattern being non-uniform. Both of these phenomenons are undesirable in a compression system, as they lead to reduced efficiency and can potentially damage system components. Therefore, the presence of one or both of these is considered as unstable operation for the system.

Two different strategies can be used to overcome the problem of surge and rotating stall: avoidance and active control. In an avoidance scheme the controller uses feedback to ensure that the system never enters an operating point in which either of the phenomenons can appear. In a control perspective this implies to ensure operation in the open loop stable region. The active control scheme is fundamentally different from the avoidance in the sense that feedback is used to stabilize the closed loop system rather than ensuring operation in some predefined region. This implies that active control gives the opportunity to operate in the open loop unstable region of the system, as opposed to the avoidance scheme.

Mass flow through the system is a variable appearing repeatedly for feedback in control of compression systems. However, real time measurement of this variable is, to the best of our knowledge, troublesome. These sensors tend to be slow or unreliable, which makes them unsuitable for feedback in a dynamic system. This motivates the development of a mass flow observer for these systems. Some previous results on the subject can be found in [1], [2], [3] and [4].

For a more comprehensive description of surge and stall phenomena and control, the reader is referred to [5], [6], [7] and the references therein.

\section{BACKGROUND}

For observer design we will use an observer based on the circle criterion, [8], [3], [9], [10]. This observer applies to systems of the form

$$
\begin{aligned}
\dot{x} & =A x+G \gamma(v)+\psi(u, y) \\
y_{c} & =C x, v=H x
\end{aligned}
$$

where $\gamma$ is a nonlinear vector function of system states, $\psi$ is a vector of measurable signals, $u$ is system input (assumed to be an available signal), $y=y(t, x, u)$ is available measurements and $y_{c}$ is available measurements that is a linear mapping of system states (typically available measurements of system states). Note that $y_{c}$ is a subset of $y$. The observer

$$
\begin{aligned}
\dot{\hat{x}} & =A \hat{x}+K_{1}\left(\hat{y}_{c}-y_{c}\right)+G \gamma(w)+\psi(u, y) \\
\hat{y}_{c} & =C \hat{x}, w=H \hat{x}+K_{2}\left(\hat{y}_{c}-y_{c}\right)
\end{aligned}
$$

was suggested by [3] for this system, where $K_{1}$ and $K_{2}$ are gains of linear injection terms. Defining the estimate error $e=$ $x-\hat{x}$ and using $V=e^{T} P e$ where $P=P^{T}>0$, it was shown that the time derivative of $V$ can be upper bounded by $\dot{V} \leq-e^{T} Q_{1} e-2(v-w)^{T} Q_{2}(\gamma(v)-\gamma(w))$ when the linear matrix inequality (LMI)

$$
\begin{aligned}
& {\left[\begin{array}{l}
P\left(A+K_{1} C\right)+\left(A+K_{1} C\right)^{T} P+Q_{1} \\
G^{T} P+Q_{2}\left(H+K_{2} C\right)
\end{array}\right.} \\
& \left.P G+\left(H+K_{2} C\right)^{T} Q_{2}\right] \leq 0
\end{aligned}
$$

is satisfied ${ }^{1}$. Hence, the equilibrium $e=0$ is exponentially stable if

$$
(v-w)^{T} Q_{2}(\gamma(v)-\gamma(w)) \geq 0,
$$

$Q_{1}>0$ and the LMI (3) has a solution. Choosing $Q_{2}=I$, a sufficient condition for (4) is $\frac{\partial \gamma(v)}{\partial v}+\left(\frac{\partial \gamma(v)}{\partial v}\right)^{T} \geq 0 \forall v$. Alternatively, $Q_{2}$ can be block diagonal with the function $\gamma$ satisfying the multivariable sector condition for the corresponding blocks [8].

For reduced order observer design, system (1) is rewritten in the form

$$
\begin{aligned}
& \dot{x}_{y}=A_{y} x_{0}+G_{y} \gamma\left(H_{y} x_{y}+H_{0} x_{0}\right)+\psi_{y}(u, y) \\
& \dot{x}_{0}=A_{0} x_{0}+G_{0} \gamma\left(H_{y} x_{y}+H_{0} x_{0}\right)+\psi_{0}(u, y)
\end{aligned}
$$

\footnotetext{
${ }^{1}$ Note that this is an LMI in $P, Q_{1}, Q_{2}, R_{1}=P K_{1}$ and $R_{2}=Q_{2} K_{2}$, where the observer gains are calculated from $K_{1}=P^{-1} R_{1}$ and $K_{2}=$ $Q_{2}^{-1} R_{2}$ after solving the LMI. Existence of $P^{-1}$ follows from $P>0$, and existence of $Q_{2}^{-1}$ can be guaranteed by requiring $Q_{2}>0$ when solving the LMI.
} 


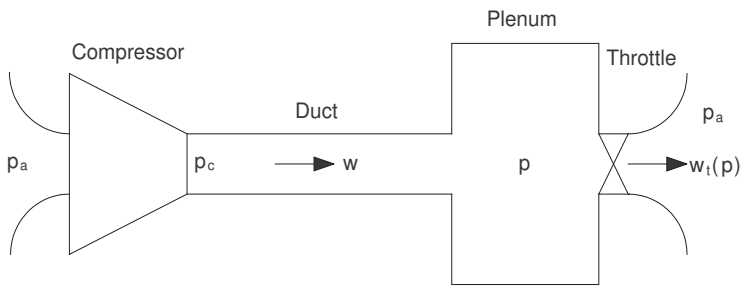

Fig. 1. Compression system

where $x_{y}$ and $x_{0}$ represents measurable and unmeasurable states respectively. Then a new variable, $\chi=x_{0}+K x_{y}$, is defined as the basis for observer design. The observer

$$
\begin{aligned}
\dot{\hat{\chi}} & =A_{\chi} \hat{\chi}+G_{\chi} \gamma\left(H_{0} \hat{\chi}+K_{\chi} x_{y}\right)+\psi_{\chi}(u, y) \\
\hat{x}_{0} & =\hat{\chi}-K x_{y}
\end{aligned}
$$

where $A_{\chi}=\left(A_{0}+K A_{y}\right), G_{\chi}=\left(G_{0}+K G_{y}\right), K_{\chi}=$ $\left(H_{y}-H_{0} K\right)$ and $\psi_{\chi}(u, y)=\psi_{0}(u, y)+K \psi_{y}(u, y)-$ $\left(A_{0}+K A_{y}\right) K x_{y}$ was suggested in [3] for this system. Analogous to the full order case, defining $e_{0}=x_{0}-\hat{x}_{0}$ and using $V\left(e_{0}\right)=e_{0}^{T} P_{0} e_{0}$ with $P_{0}=P_{0}^{T}$, an upper bound $\dot{V}\left(e_{0}\right) \leq-e_{0}^{T} Q_{1} e_{0}-2\left(v_{0}-w_{0}\right)^{T} Q_{2}\left(\gamma\left(v_{0}\right)-\gamma\left(w_{0}\right)\right)$ was found when the LMI

$$
\begin{aligned}
& {\left[\begin{array}{l}
P_{0}\left(A_{0}+K A_{y}\right)+\left(A_{0}+K A_{y}\right)^{T} P_{0}+Q_{1} \\
\left(G_{0}+K G_{y}\right)^{T} P_{0}+Q_{2} H_{0}
\end{array}\right.} \\
& \left.P_{0}\left(G_{0}+K G_{y}\right)+H_{0}^{T} Q_{2}\right] \leq 0
\end{aligned}
$$

is satisfied. Following the arguments and requirements of the full order case, $e_{0}=0$ is exponentially stable. Furthermore, it was shown in [3] that a reduced-order observer exists if there is a solution to the full order problem.

\section{COMPRESSION SYSTEM MODEL}

A classical result in the field of compressor surge modeling is the model of Greitzer [11], which covers a basic compression system consisting of a compressor, a plenum volume, in-between ducting and a throttle as shown in Fig. 1. In [12] the authors extended the Greitzer model to also incorporate variable impeller speed. A similar model was derived in [13] using an approach based on energy analysis, which is the model used here.

Consider a compression system in which a centrifugal compressor supplies compressed gas to a duct discharging into a plenum volume, from which the compressed gas discharges over a throttle. A model for this system can be taken as

$$
\begin{aligned}
\dot{p} & =\frac{c_{a}^{2}}{V}\left(w-w_{t}\left(A_{t \%}, p\right)\right) \\
\dot{w} & =\frac{A}{L}\left(p_{c}(w, \omega)-p\right) \\
\dot{\omega} & =\frac{1}{J}\left(\tau_{d}-\tau_{c}(w, \omega)\right) .
\end{aligned}
$$

where $p$ is the plenum pressure, $w$ is the duct mass flow and $\omega$ is impeller speed. The various functions represents throttle mass flow $w_{t}\left(A_{t}, p\right)$, total pressure downstream compressor $p_{c}(w, \omega)$ and torque experienced by impeller due to compressor fluid flow $\tau_{c}(w, \omega)$. Variable throttle flow through area $A_{t \%}$ and drive torque $\tau_{d}$ are considered as inputs to this system. Furthermore, the various constants represent speed of sound at ambient conditions $c_{a}$, volume of plenum $V$, cross section of duct $A$, length of duct $L$ and inertia of rotating parts $J$.

Pressure dynamics is derived by computing the mass balance of the plenum volume assuming isentropic conditions and uniform pressure. Mass flow dynamics is derived by computing the momentum balance of the duct connecting compressor and plenum assuming incompressible one dimensional flow in the duct and compressor. Moreover, dynamic effects related to the compressor stage are assumed small, leaving total pressure downstream the compressor as a pure mapping from mass flow and impeller speed. Impeller speed dynamics is derived by calculating the angular momentum balance.

Models for throttle mass flow and compressor torque are taken as

$$
\begin{aligned}
w_{t}\left(A_{t \%}, p\right) & =k_{t}\left(A_{t \%}\right) \operatorname{sign}\left(p-p_{a}\right) \sqrt{\left|p-p_{a}\right|} \\
\tau_{c}(w, \omega) & =k_{c}|w| \omega,
\end{aligned}
$$

where $p_{a}$ is ambient constant pressure, $k_{t}$ is a function reflecting the throttle opening and $k_{c}$ is a compressor torque constant. The model of throttle mass flow is slightly modified relative to [13]. The modification involves including the possibility of variable area throttle and negative mass flow through the valve. To this end the throttle mass flow is assumed to be symmetrical terms of positive and negative mass flow as a function of pressure across the devise. The input $A_{t \%}$ is assumed to take on values in the range $0-100$ (or equivalently $0-1$ ) corresponding to minimum and maximum flow through area respectively. Furthermore, the function $k_{t}\left(A_{t}\right)$ is assumed positive for all valid values of $A_{t \%}$ (possibly zero for $A_{t \%}=0$, when this might represent a completely closed throttle).

For notational convenience of subsequent analysis, system states, inputs, constants and functions are redefined.

Definition 1: $x_{1}=p, x_{2}=w, x_{3}=\omega, x=$ $\left[\begin{array}{ccc}x_{1} & x_{2} & x_{3}\end{array}\right]^{T}, u_{1}=A_{t \%}, u_{2}=\tau_{d}, u=\left[\begin{array}{ll}u_{1} & u_{2}\end{array}\right]^{T}$, $k_{1}=\frac{c_{a}^{2}}{V}, k_{2}=\frac{A}{L}, k_{3}=\frac{1}{J}, k_{4}=k_{t}, k_{5}=p_{a}, k_{6}=$ $k_{c}, f_{1}\left(u_{1}, x_{1}\right)=w_{t}\left(A_{t \%}, p\right), f_{2}\left(x_{2}, x_{3}\right)=p_{c}(w, \omega)$ and $f_{3}\left(x_{2}, x_{3}\right)=\tau_{c}(w, \omega)$.

Furthermore, the approximation

$$
f_{3}\left(x_{2}, x_{3}\right) \approx k_{6} \tanh \left(\zeta x_{2}\right) x_{2} x_{3}
$$

will be used for (10) when differentiation of $f_{3}$ with respect to $x_{2}$ is required. It can be shown that $\lim _{\zeta \rightarrow \infty} k_{6} \tanh \left(\zeta x_{2}\right) x_{2} x_{3} \quad \rightarrow \quad k_{6}\left|x_{2}\right| x_{3} \quad$ and $\frac{\partial f_{3}\left(x_{2}, x_{3}\right)}{\partial x_{2}}=k_{6} k_{7}(t) x_{3}$ where

$k_{7}(t)=\zeta x_{2}\left(1-\tanh ^{2}\left(\zeta x_{2}\right)\right)+\tanh \left(\zeta x_{2}\right) \in[-1.2,1.2]$. 
The functions of Definition 1 can be shown to satisfy

$$
\begin{aligned}
& (a-b)\left(f_{1}\left(u_{1}, a\right)-f_{1}\left(u_{1}, b\right)\right) \geq 0 \\
& (a-b)\left(f_{2}\left(x_{2}, a\right)-f_{2}\left(x_{2}, b\right)\right) \geq 0 \\
& (a-b)\left(f_{3}\left(x_{2}, a\right)-f_{3}\left(x_{2}, b\right)\right) \geq 0
\end{aligned}
$$

by using (9), (10) and the fact that downstream pressure is strictly increasing in impeller speed, [5].

For observer design we assume that plenum pressure, downstream compressor pressure, and impeller speed are measurable and that system inputs are available. From Definition 1 it then follows that

$$
\begin{aligned}
y & =\left[\begin{array}{lll}
x_{1} & x_{3} & f_{2}
\end{array}\right]^{T}=\left[\begin{array}{lll}
p & \omega & p_{c}
\end{array}\right]^{T}, \\
y_{c} & =x_{y}=\left[\begin{array}{ll}
x_{1} & x_{3}
\end{array}\right]^{T}=\left[\begin{array}{ll}
p & \omega
\end{array}\right]^{T}, \\
C & =\left[\begin{array}{lll}
1 & 0 & 0 \\
0 & 0 & 1
\end{array}\right],
\end{aligned}
$$

where $y_{c}$ and $x_{y}$ relates to the full order and reduced order observer respectively.

\section{FULL ORDER OBSERVER}

Even though the observer design for this system was motivated by the lack of a mass flow measurement, a full state observer might also offer disturbance rejection for the measured plenum pressure and impeller speed as well as incorporating more model information for the estimate of mass flow. Therefore, we start by deriving a full state observer for (8).

Proposition 2: If the LMI (3) is solvable for $Q_{2}=$ $\operatorname{diag}\left\{q_{21}, q_{22}, q_{22}\right\}, H=I_{3 \times 3}, C$ from (18),

$$
A=\left[\begin{array}{ccc}
0 & \frac{c_{a}^{2}}{V} & 0 \\
-\frac{A}{L} & -c_{2} & 0 \\
0 & -c_{3} & 0
\end{array}\right] \text { and } G=\left[\begin{array}{ccc}
-1 & 0 & 0 \\
0 & 1 & 0 \\
0 & 0 & -1
\end{array}\right] \text {, }
$$

where $q_{2 i} \geq 0, c_{2}>0$ and $c_{4} c_{2}>\frac{3 k_{c}^{2}}{J^{2} 25}$, then (2) with

$$
\begin{aligned}
\gamma(w) & =\left[\begin{array}{c}
c_{a}^{2} \\
V \\
\left.c_{2} w_{2}+A_{t}, w_{1} w_{3}\right) \\
\frac{1}{J} \tau_{c}\left(w_{2}, w_{3}\right)-c_{3} w_{2}+c_{4} w_{3}^{3}
\end{array}\right], \\
\psi(u, y) & =\left[\begin{array}{c}
0 \\
\frac{A}{L} p_{c}-c_{3} \omega \\
\frac{1}{J} \tau_{d}+c_{4} \omega^{3}
\end{array}\right],
\end{aligned}
$$

is an observer for (8)-(10).

Proof: Using Definition 1 the model (8) can be formulated in the form (1) with

$$
\begin{aligned}
& \gamma=\left[\begin{array}{c}
k_{1} f_{1}\left(u_{1}, x_{1}\right) \\
c_{2} x_{2}+\alpha_{2}(y) \\
k_{3} f_{3}\left(x_{2}, x_{3}\right)-c_{3} x_{2}-\alpha_{3}(y)
\end{array}\right], \\
& 0 \\
& \psi=\left[\begin{array}{c}
0 \\
k_{2} f_{2}\left(x_{2}, x_{3}\right)-\alpha_{2}(y) \\
k_{3} u-\alpha_{3}(y)
\end{array}\right],
\end{aligned}
$$

and the matrixes from Proposition 2, by adding and subtracting $c_{2} x_{2}, \alpha_{2}(y)$ and $c_{3} x_{2}, \alpha_{3}(y)$ in the $\dot{x}_{2}$ and $\dot{x}_{3}$ equations respectively. Adding and subtracting linear terms in the unknowns and nonlinear terms of known signals is done to get greater freedom when evaluating the sector property (4) for $\gamma$. Defining $v=\left(v_{1}, v_{2}, v_{3}\right)=\left(x_{1}, x_{2}, x_{3}\right)$, it follows from (1b) that $H$ is defined as in Proposition 2. For notational convenience we now define the vectors $z=v-w$ and $\varphi(t, v, w)=\gamma(t, v)-\gamma(t, w)$, where the time argument comes from using $u_{1}$ in $\gamma$. Furthermore, by choosing $Q_{2}=$ $\operatorname{diag}\left\{q_{21}, q_{22}, q_{22}\right\}$ we can rewrite

$$
\begin{aligned}
z^{T} Q_{2} \varphi(t, v, w) & =q_{21} z_{1} \varphi_{1}\left(t, v_{1}, w_{1}\right) \\
& +q_{22} z_{[2,3]}^{T} \varphi_{[2,3]}\left(v_{[2,3]}, w_{[2,3]}\right)
\end{aligned}
$$

where $v_{[2,3]}=\left(v_{2}, v_{3}\right), w_{[2,3]}=\left(w_{2}, w_{3}\right), z_{[2,3]}=v_{[2,3]}-$ $w_{[2,3]}$ and $\varphi_{[2,3]}$ is a vector consisting of the two last components of $\varphi(t, v, w)$. This implies that $z_{1}=v_{1}-w_{1}$ and that $\varphi_{1}\left(t, v_{1}, w_{1}\right)$ is a scalar being equal to the first component of $\varphi(t, v, w)$. From (19) and (13) it follows that

$$
q_{21} z_{1} \varphi_{1}\left(t, v_{1}, w_{1}\right)=k_{1} q_{21} z_{1}\left(f_{1}\left(u_{1}, v_{1}\right)-f_{1}\left(u_{1}, w_{1}\right)\right) \geq 0
$$

for $q_{21} \geq 0$ since $k_{1}>0$. For $q_{22} z_{[2,3]}^{T} \varphi_{[2,3]}\left(v_{[2,3]}, w_{[2,3]}\right)$ we evaluate the multivariable sector property $\frac{\partial \gamma_{[2,3]}\left(v_{[2,3]}\right)}{\partial v_{[2,3]}}+$ $\left(\frac{\partial \gamma_{[2,3]}\left(v_{[2,3]}\right)}{\partial v_{[2,3]}}\right)^{T} \geq 0$, where $\gamma_{[2,3]}$ refers to the lower two elements of (19). The upper left determinants of this matrix are found by using (19), (11) and (12), and give the conditions

$$
\begin{aligned}
0 & <2 c_{2} \\
0 & <4 c_{2}\left(k_{3} k_{6}\left|v_{2}\right|-\frac{\partial \alpha_{3}\left(v_{3}\right)}{\partial v_{3}}\right) \\
& -\left(k_{3} k_{6} k_{7}(t) v_{3}+\frac{\partial \alpha_{2}\left(v_{3}\right)}{\partial v_{3}}-c_{3}\right)^{2} .
\end{aligned}
$$

From (21) it is clear that we must require $c_{2}>0$. Furthermore, by choosing $\alpha_{2}\left(v_{3}\right)=c_{3} v_{3}$ and $\alpha_{3}\left(v_{3}\right)=-c_{4} v_{3}^{3}$ the inequality (22) is rewritten

$$
4 c_{2} k_{3} k_{6}\left|v_{2}\right|+\left(12 c_{2} c_{4}-k_{3}^{2} k_{6}^{2} k_{7}^{2}(t)\right) v_{3}^{2}>0 .
$$

Since $k_{7}(t)$ is in the range of $-\frac{6}{5}$ to $\frac{6}{5},(12)$, the inequality (23) is satisfied if $c_{4} c_{2}>\frac{3 k_{3}^{2} k_{6}^{2}}{25}$. Notice that the constant $c_{3}$ can be chosen as any constant (positive, negative and even zero). These choices for $Q_{2}, \alpha_{2}\left(v_{3}\right), \alpha_{3}\left(v_{3}\right)$ and the requirements for the gains, ensures validity the multivariable sector property $z^{T} Q_{2} \varphi(v, w) \geq 0$. Inserting for $\alpha_{2}$ and $\alpha_{3}$ in (19) and (20) gives the $\gamma$ and $\psi$ of Proposition 2.

\section{REDUCED-ORDER OBSERVER FOR MASS FLOW}

Based on the previous analysis of the full order observer, we now derive a reduced-order observer for mass flow. As already mentioned, a reduced-order observer exists if the full order observer exists, [3]. 
Proposition 3: If the LMI (7) is solvable for $Q_{2}=$ $\operatorname{diag}\left\{q_{21}, q_{22}, q_{22}\right\}, C$ from (3) and

$$
\begin{aligned}
& A_{y}=\left[\begin{array}{c}
\frac{c_{a}^{2}}{V} \\
-c_{3}
\end{array}\right], A_{0}=-c_{2} \\
& G_{y}=\left[\begin{array}{ccc}
-1 & 0 & 0 \\
0 & 0 & -1
\end{array}\right], G_{0}=\left[\begin{array}{lll}
0 & 1 & 0
\end{array}\right] \\
& H_{y}=\left[\begin{array}{ll}
1 & 0 \\
0 & 0 \\
0 & 1
\end{array}\right], H_{0}=\left[\begin{array}{l}
0 \\
1 \\
0
\end{array}\right]
\end{aligned}
$$

where $q_{2 i} \geq 0, c_{2}>0$ and $c_{4} c_{2}>\frac{3 k_{c}^{2}}{J^{2} 25}$, then (6) with

$$
\begin{aligned}
\gamma(w) & =\left[\begin{array}{c}
\frac{c_{a}^{2}}{V} w_{t}\left(A_{t \%}, w_{1}\right) \\
c_{2} w_{2}+c_{3} w_{3} \\
\frac{1}{J} \tau_{c}\left(w_{2}, w_{3}\right)-c_{3} w_{2}+c_{4} w_{3}^{3}
\end{array}\right] \\
\psi_{y} & =\left[\begin{array}{c}
0 \\
k_{3} \tau_{d}+c_{4} \omega^{3}
\end{array}\right] \\
\psi_{0} & =\frac{A}{L} p_{c}-\frac{A}{L} p-c_{3} \omega
\end{aligned}
$$

is an observer for $(8 b)$.

Proof: The model (8) can be formulated in the form (5) with

$$
\begin{aligned}
\gamma & =\left[\begin{array}{c}
k_{1} f_{1}\left(x_{1}\right) \\
c_{2} x_{2}+\alpha_{2}(y) \\
k_{3} f_{3}\left(x_{2}, x_{3}\right)-c_{3} x_{2}-\alpha_{3}(y)
\end{array}\right] \\
\psi_{y} & =\left[\begin{array}{c}
0 \\
k_{3} u-\alpha_{3}(y)
\end{array}\right] \\
\psi_{0} & =k_{2} f_{2}\left(x_{2}, x_{3}\right)-k_{2} x_{1}-\alpha_{2}(y)
\end{aligned}
$$

and the matrixes from Proposition 3 by adding and subtracting $c_{2} x_{2}, \alpha_{2}(y)$ and $c_{3} x_{2}, \alpha_{3}(y)$ in the $\dot{x}_{2}$ and $\dot{x}_{3}$ equations respectively. Since $\gamma$ is the same as that for the full order case, (19), it is known that choosing $\alpha_{2}\left(v_{3}\right)=c_{3} v_{3}$ and $\alpha_{3}\left(v_{3}\right)=-c_{4} v_{3}^{3}$ with $c_{2}>0, c_{3} \in \mathbb{R}, c_{2} c_{4}>$ $\frac{k_{3}^{2} k_{6}^{2}}{12}$ and $Q_{2}=\operatorname{diag}\left(q_{21}, q_{22}, q_{22}\right), q_{2 i} \geq 0$ guarantees $z^{T} Q_{2}(\gamma(v)-\gamma(w)) \geq 0$ (where $\left.z=v-w\right)$.

Remark 4: Proposition 3 can alternatively be formulated by replacing $A_{0}=0, G_{0}=\left[\begin{array}{lll}0 & 0 & 0\end{array}\right]$ and $\psi_{0}=k_{2} p_{c}-k_{2} p$. This follows from the proof of Proposition 3, since $\gamma$, (19), will be unchanged.

\section{Simulation}

The compressor map used for simulations is the same as used in [14]. This is a map based on measurement data, for which third order approximations in both compressor speed and mass flow are done to make the map continuous in these variables. Denoting equilibria states by superscript $e$, it can be seen from (8) it can be seen that a equilibrium satisfy

$$
\begin{aligned}
w^{e} & =k_{t} \sqrt{p^{e}-p_{a}} \\
p^{e} & =p_{c}\left(w^{e}, \omega^{e}\right) \\
\tau_{d}^{e} & =k_{c} w^{e} \omega^{e}
\end{aligned}
$$

where in all practical cases the desired equilibrium involves positive valued states and a higher plenum than ambient

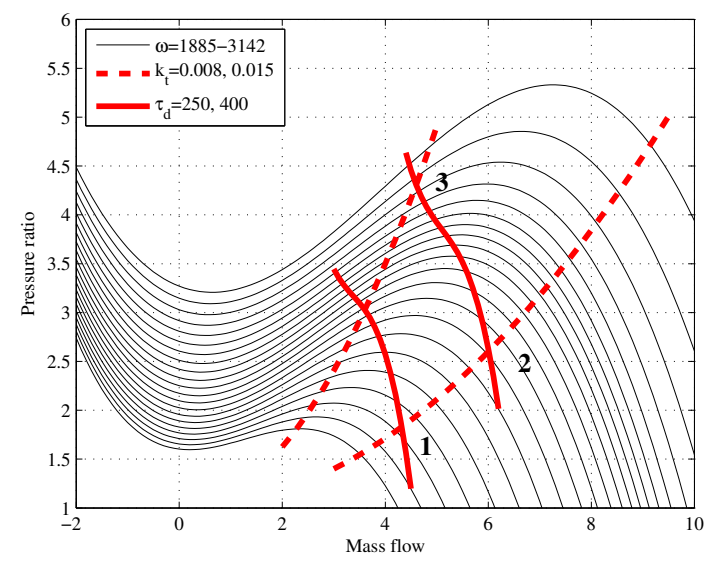

Fig. 2. Equilibrium points represented in compressor map

pressure. Using (24) and (25), the throttle characteristics can be plotted in the compressor map. This is illustrated in Fig. 2 for two different throttle openings (represented by different $k_{t}$ 's), where the intersection of compressor and throttle characteristics constitutes the possible equilibria of the system. This means that freedom to choose a desired operating point for the compression system using only the drive torque as actuator is limited to some point on the throttle characteristics.

Using (25) and (26), the torque characteristic can be plotted in the compressor map. This is illustrated in Fig. 2 for two constant torque inputs of different amplitude. The system equilibrium is then given by the intersection of throttle and torque characteristic.

Simulations will go through a scenario in which the system is initially operating in point 1 of Fig. $2\left(k_{t}=0.015\right.$ and $\left.\tau_{d}=250\right)$. The system is then driven to operating point 2 ( $k_{t}=0.015$ and $\left.\tau_{d}=400\right)$ after 100 seconds, which involves a change of torque input. The system is driven to operating point $3\left(k_{t}=0.008\right.$ and $\left.\tau_{d}=400\right)$ after 600 seconds, which involves a change in the throttle opening. Change of torque and throttle opening is done with a step change of the parameter in question, in series with a first order filter (time constant of $T=10)$.

For the described scenario, the throttle openings constitute equilibrium points for which the compressor characteristics has a negative and positive slope in $w$. From the literature it is well known that a negative slope implies stable equilibria. For positive slope however, the system need not be stable and surge can occur.

The open loop response is shown in Fig. 3. This simulation shows stable behavior for the first two operating points, before eventually entering surge in the last operating point. From the plot of compressor speed, it seems like this state is not oscillating. However, a closer examination reveals relative small oscillation also for this state.

Simulation of the full order observer from Proposition 2 is 

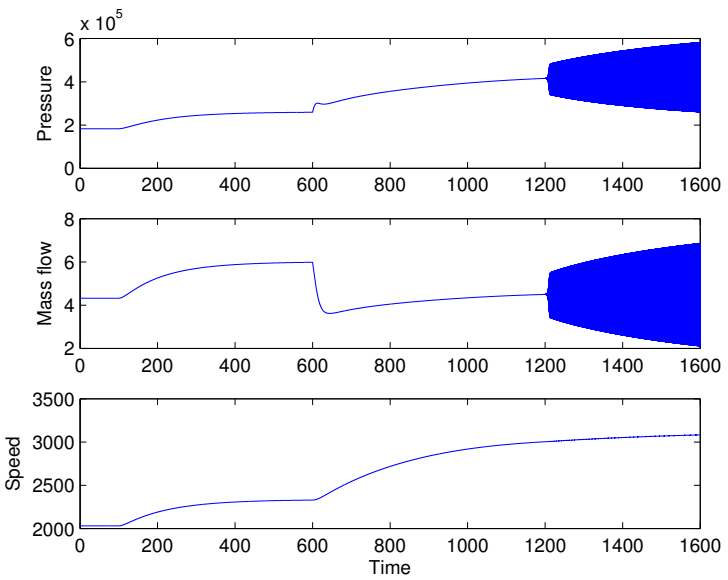

Fig. 3. Simulation of system states
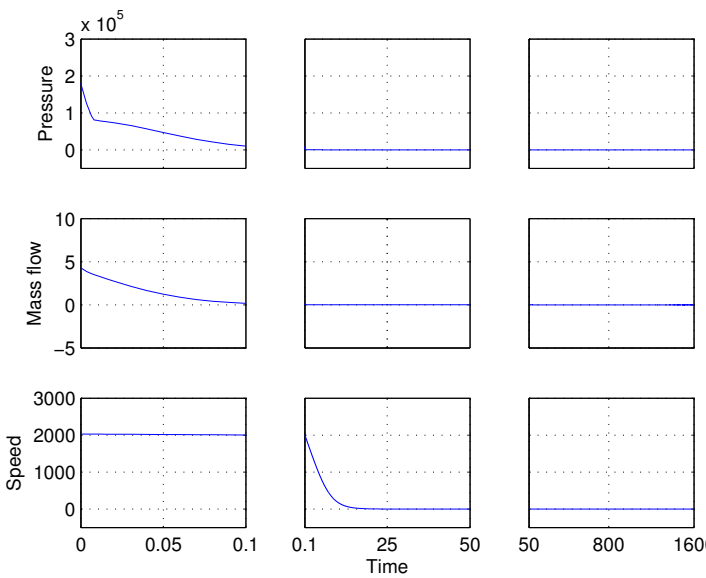

Fig. 4. Error variables for the full order observer

shown in Fig. 4, using observer gains

$$
\begin{aligned}
K_{1} & =\left[\begin{array}{cc}
9.7 \cdot 10^{-7} & -3.1 \cdot 10^{-14} \\
-1.7 \cdot 10^{-11} & 8.0 \cdot 10^{-19} \\
-2.6 \cdot 10^{-17} & -3.0 \cdot 10^{-8}
\end{array}\right], \\
K_{2} & =\left[\begin{array}{ll}
10^{-3} & 10^{-1} \\
10^{-8} & 10^{-6} \\
10^{-5} & 10^{-3}
\end{array}\right],
\end{aligned}
$$

$c_{2}=10^{2}, c_{3}=10^{-4}$ and $c_{4}=10^{-8}$. The figure shows observer error, $e=x-\hat{x}$, and is divided in three columns. These columns represent different time intervals of the simulation, and the division is done to show the convergence for the various estimates. The first two columns shows that pressure and mass flow estimates converges relatively fast with respect to impeller speed. The third column shows that the observer error remains zero, also when entering surge.

Simulation of the reduced order observer from Proposition 2 is shown in Fig. 5, using control gains

$$
K=\left[\begin{array}{ll}
-4.7 \cdot 10^{-8} & 1.2 \cdot 10^{-18}
\end{array}\right]
$$

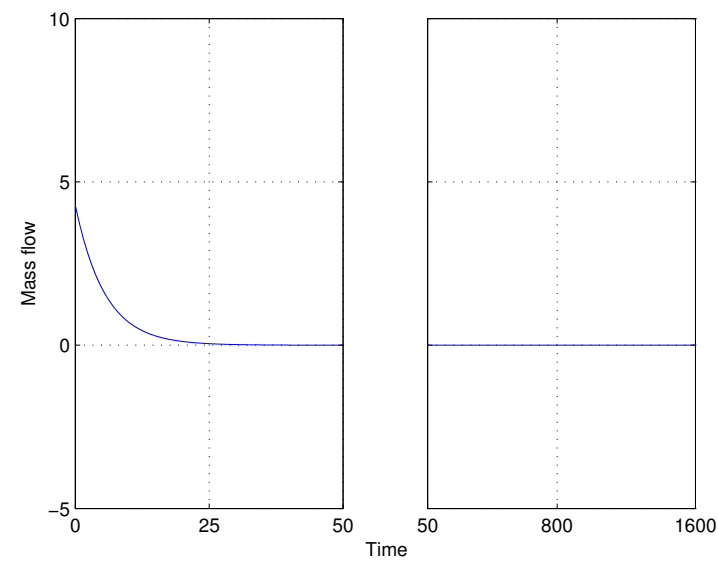

Fig. 5. Error variables for the reduced order observer

and $c_{i}$ 's equal to those of the full order observer. Also here the time axis is split up to make the convergence visible. As can be seen from Fig. 4 and Fig. 5, the convergence of the mass flow estimate for the reduced order observer is in the range of that for the full order observer.

Measurement noise was simulated by adding normally distributed random numbers to the measurements. These random numbers where in the range \pm 3000 for the measurement of $y_{1}$ and $y_{3}$, and \pm 30 for $y_{2}$. The result is shown in Fig. 6 and Fig. 7 for the full and reduced order observer respectively. From Fig. 6 it can be seen that the full order observer suppresses noise of both pressure and impeller speed measurements, and the noise on the mass flow estimate is in the range of $1 \%$ of the actual value. The noise on the pressure estimate is only damped slightly with respect to the measurement, whereas the noise on the speed estimate is reduced by a factor of $15-20$ relative to the noise on the measurement. From Fig. 7 it can be seen that the noise on the mass flow estimate for the reduced order observer is in the range of $10 \%$ of the actual value. Hence, a decade larger than that of the full order observer.

\section{CONCLUDING REMARKS}

The inequality (3) is an LMI in $P, Q_{1}, Q_{2} R_{1}=P K_{1}$ and $R_{2}=Q_{2} K_{2}$, where $R_{1}$ and $R_{2}$ are defined to make the inequality a LMI. Hence, the control gains are calculated after solving the LMI $\left(K_{1}=P^{-1} R_{1}\right.$ and $\left.K_{2}=Q_{2}^{-1} R_{2}\right)$, and no explicit criteria can be posted for these gains when solving the LMI. To get small control gains, which is desirable for disturbance rejection, we generally desire large $P$ and $Q_{2}$, and small $R_{1}$ and $R_{2}$. Since $P$ and $Q_{2}$ are positive definite, these can be made large by minimization of a linear objective under LMI constraints (by weighing the diagonal elements by negative values). The $R_{i}$ 's on the other hand, need not be sign definite and quadratic. An optimization criteria will therefore involve minimizing some weight norm of their components under LMI constraints. However, this is not possible with the LMI Lab in MATLAB. The same problem will arise for the 

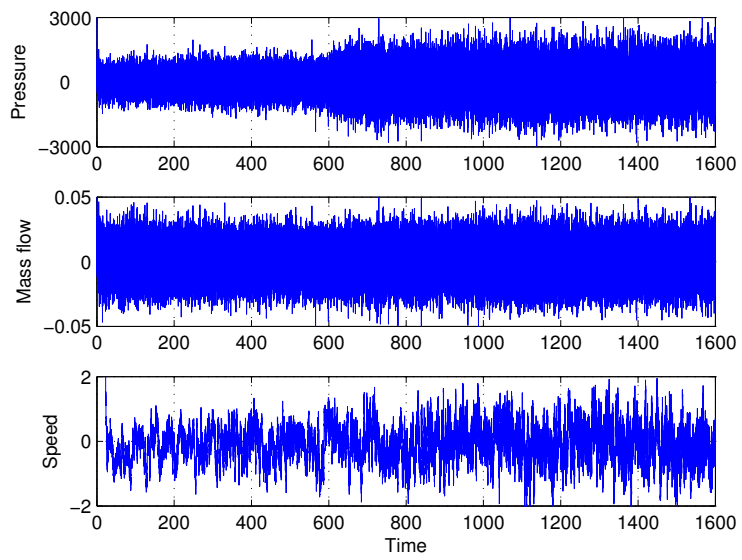

Fig. 6. Error variables for the full order observer with measurement noise.

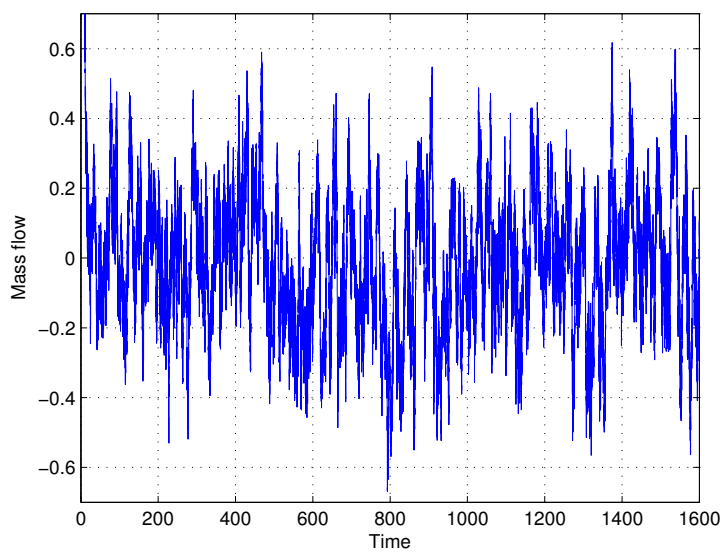

Fig. 7. Error variables for the reduced order observer with measurement noise.

inequality (8), when this is an LMI in $P_{0}, Q_{1}, Q_{2}$ and $R=$ $P_{0} K$.

Tuning of the observer gains for Proposition 2 was therefore done in several steps. The gain $K_{1}$ was derived by pole placement for $A+K_{1} C$. The gain $K_{2}$ was derived by a matrix structure of corresponding dimensions multiplied by a scalar, where the structure was chosen such that $K_{2, \text { structure }} y_{c}^{e}$ would be in the same order of magnitude as $x^{e}$ (where the superscript $e$ refers to system equilibrium). The resulting $K_{1}$ and $K_{2}$ was then checked with (3) to see if they where possible solutions. A similar approach was taken when choosing the gain $K$ for the reduced order observer.

From the simulations where measurement noise has been added, Fig. 6 and Fig. 7, it can be seen that the full order observer offers better disturbance rejection for mass flow than the reduced order observer, even though the convergence rate for these are in the same range. One possible explanation for this can be found in the $\psi$ 's for the respective observers. These functions enter the integrator of the observers directly, without the chance of reducing their amplitude by observer gains. They are given by $\psi(u, y)=k_{2} y_{3}-c_{3} y_{2}$ for the mass flow dynamics of the full order observer and $\psi_{\chi}(u, y)=$ $\underbrace{k_{2} y_{3}-c_{3} y_{2}}_{\psi}-k_{2} y_{1}+K \psi_{y}(u, y)-\left(A_{0}+K A_{y}\right) K x_{y}$ for the reduced order observer. From these equations it is clear that $\psi_{\chi}$ represents a more noisy signal than $\psi$, since $\psi$ is contained in $\psi_{\chi}$ in addition to other noisy signals. Moreover, the $\psi$ 's will be the main limiting factor for disturbance rejection, since the noise that enters through these can not be reduced by control gains (some of the signals that enter through $\psi_{\chi}$ can be reduced by $K$, but not all).

\section{REFERENCES}

[1] N. A. Chaturvedi and S. P. Bhat, "Output-feedback semiglobal stabilization of stall dynamics for preventing hysteresis and surge in axialflow compressors," Control Systems Technology, IEEE Transactions on, vol. 14, no. 2, pp. 301-307, March 2006.

[2] K. O. Boinov, E. A. Lomonova, A. J. A. Vandenput, and A. Tyagunov, "Surge control of the electrically driven centrifugal compressor," Industry Applications Conference, vol. 4, pp. 2887-2894, October 2005.

[3] M. A. and P. V. Kokotović, "Nonlinear observers: a circle criterion design and robustness analysis," Automatica, vol. 37, no. 12, pp. 19231930, 2001.

[4] O. O. Badmus, S. Chowdhury, and C. N. Nett, "Nonlinear control of surge in axial compression systems," Automatica, vol. 32, no. 1, pp. 59-70, January 1996.

[5] J. T. Gravdahl and O. Egeland, Compressor surge and rotating stall: modeling and control, ser. Advances in Industrial Control. SpringerVerlag, 1999.

[6] F. Willems and B. de Jager, "Modeling and control of compressor flow instabilities," IEEE Control Systems Magazine, vol. 19, no. 5, pp. 8-18, 1999.

[7] J. D. Paduano and E. M. A. H. Epstein, "Compression system stability and active control," Annual Review of Fluid Mechanics, vol. 33, no. 1, pp. 491-517, 2001.

[8] X. Fan and M. Arcak, "Observer design for systems with multivariable monotone nonlinearities," Systems \& Control Letters, vol. 50, pp. 319 330, 2003.

[9] M. Arcak and P. Kokotović, "Observer-based control of systems with slope-restricted nonlinearities," Automatic Control, IEEE Transactions on, vol. 46, no. 7, pp. 1146-1150, 2001.

[10] — "Feasibility conditions for circle criterion designs," Systems and Control Letters, vol. 42, no. 5, pp. 405-412, 2001.

[11] E. M. Greitzer, "Surge and rotating stall in axial flow compressors, part i: Theoretical compression system model," Journal of Engineering for Power, vol. 98, pp. 190-198, 1976.

[12] D. A. Fink, N. A. Cumpsty, and E. M. Greitzer, "Surge dynamics in free-spool centrifugal compressor system," Journal of Turbomachinery, vol. 114, pp. 321-332, 1992.

[13] J. T. Gravdahl, F. Willems, B. de Jager, and O. Egeland, "Modeling of surge in variable speed centrifugal compressors: Experimental validation," AIAA Journal of Propulsion and Power, vol. 20, no. 5, pp. 849-857, September 2004.

[14] J. T. Gravdahl, O. Egeland, and S. O. Vatland, "Active surge control of centrifugal compressors using drive torque," in Procedings of the 40th IEEE Conference on Desition and Control, December 2001. 